\title{
A systematic review on the effect of bevacizumab in exudative age-related macular degeneration
}

\author{
Jan S. A. G. Schouten • Ellen C. La Heij • \\ Carroll A. B. Webers • Igor J. Lundqvist • \\ Fred Hendrikse
}

Received: 16 April 2008 /Revised: 4 September 2008 / Accepted: 5 September 2008 / Published online: 9 October 2008

(C) The Author(s) 2008. This article is published with open access at Springerlink.com

\begin{abstract}
Aim To provide evidence for the effect of bevacizumab on visual acuity (VA) and central retinal thickness (CRT) in exudative age-related macular degeneration

Methods A systematic review of all articles of bevacizumab for exudative AMD was conducted. Articles published up to March 2008 were identified in Medline, Embase, the Cochrane Controlled Trials Register and references from included articles. Search terms were "Bevacizumab or Avastin" and "Macula* or ARMD or AMD or intra(-) vitreal or intra(-)vitreous". Three observers participated in the data retrieval and assignment of the quality scores.

Results A total of 561 articles were retrieved. Three randomised controlled trials (RCT) and 23 before-and-after studies of patients $(n=1,435)$ who had received bevacizumab were published. Inclusion criteria varied. Lack of masking was the main methodological shortcoming. These RCTs showed that bevacizumab is more effective than PDT. Bevacizumab was given intravenously or as intravitreal injection. The latter was given once, or repeatedly every 4 weeks, and with or without additional injection when a recurrence occurred, mostly based on visual acuity and/or findings from optical coherence tomography. After intravenous administration, the weighted mean change in VA was +12.8 ETDRS letters (range +11 to +14 ) and the weighted mean change for CRT was $-129 \mu \mathrm{m}$ (range -100
\end{abstract}

The authors had no commercial or proprietary interest.

J. S. A. G. Schouten $(\bowtie) \cdot$ E. C. La Heij • C. A. B. Webers •

I. J. Lundqvist $\cdot$ F. Hendrikse

Department of Ophthalmology, Maastricht University Hospital,

P.O. box 5800, 6202 AZ Maastricht, The Netherlands

e-mail: J.Schouten@MUMC.nl

E. C. La Heij

e-mail: ellen.laheij@mumc.nl to -202). For the 23 studies with intravitreal injections, the change in VA was +8.6 letters (range +2 to +26 ) and the change in CRT was $-90 \mu \mathrm{m}$ (range -46 to -190 ). The incidence of adverse events was low. The change in VA was 2.7 letters higher for studies with a higher quality vs lower quality.

Conclusion Visual acuity improves and central retinal thickness decreases in patients with exudative AMD after bevacizumab. There is no reasonable doubt that this is caused by bevacizumab. It is likely that a randomised controlled trial will show that bevacizumab is equivalent in effect to ranibizumab, which showed a change in ETDRS of +5.9 letters for occult or minimally classic $\mathrm{CNV}$ and +9.8 letters for classic $\mathrm{CNV}$ after three monthly injections in two large RCTs.

Keywords Systematic review - Bevacizumab - Exudative macular degeneration $\cdot$ Visual acuity $\cdot$ Retinal thickness

\section{Introduction}

Age-related macular degeneration (AMD) is the leading cause of severe blindness in the elderly in Western countries $[11,13]$. Exudative macular degeneration is the most severe form with the highest risk of blindness [13]. Vision loss is the result of macular haemorrhage and fluid caused by leakage from subfoveal choroidal neovascularisation (CNV), which is the hallmark of exudative AMD. Vascular endothelial growth factors (VEGF) have a key role in the formation and leakage of CNV [28]. Recently, the anti-VEGFs pegabtanib and ranibizumab became available for the treatment of exudative AMD [12, 36, 38, 43, 45]. In contrast to pegabtanib, intravitreal injections with ranibizumab led to a significant vision improvement. In fact, 
ranibizumab was the first drug to improve vision in exudative AMD, compared to interventions used earlier which only delayed progression [12, 38]. Major disadvantages of this intervention are the costs and the need to give intravitreal injections repeatedly. Bevacizumab, which is approved for the treatment of colon cancer but not for the treatment of $\mathrm{AMD}$, is closely related to ranibizumab. In contrast to pegaptanib that binds only the VEGF isoforms that are $165 \mathrm{kD}$ (VEGF165) and larger [26], both ranibizumab and bevacizumab bind and inhibit all biologically active forms of VEGF [47]. Bevacizumab is a fulllength, humanized monoclonal antibody against VEGF, whereas ranibizumab is a humanized antigen binding fragment against VEGF, and both proteins were genetically engineered from the same murine monoclonal antibody against VEGF. A major advantage of bevacizumab is its price, which is about $1-5 \%$ the price of ranibizumab. Mainly for this reason, it is now used worldwide and on a large scale off-label for the treatment of exudative AMD. Its off-label use is obviously controversial, also because the use of anti-VEGF therapy is associated with an increased risk for thromboembolic events. Until now, no systematic review has shown evidence for the therapeutic effect of bevacizumab, or its safety. In this systematic review, the effects of bevacizumab on visual acuity (VA) and central retinal thickness (CRT) are quantified, and the adverse events are reported. Moreover, the various therapeutic strategies are described.

\section{Material and methods}

\section{Searching}

The databases searched were MEDLINE, EMBASE, and the Cochrane database. The reference lists of included articles and personal files were also searched until no new articles were found. Search terms for the application in age-related macular degeneration were "Macula*" or "AMD" or "ARMD" or "intra(-)vitreous" or "intra-vitreal" in any field, and for the intervention the search terms were "Bevacizumab" or "Avastin" in any field. All years were included up to March 2008. Published articles on paper, or papers available electronically before publication on paper were included. Language restrictions were English, German, French or Dutch. Two observers, independently of each other, searched the acquired list to identify relevant articles.

\section{Selection}

Articles included were randomised controlled trials, nonrandomised controlled studies, or before-and-after studies in more than one patient. Studies with systemic or intravitreal therapy were included. Studies were excluded that did not have VA as the primary outcome, had as the primary objective to study differences between subgroups, or included also patients other than patients with exudative AMD. All articles selected by at least one observer were copied or printed. The ultimate selection was based on a discussion between observers.

Data abstraction and validity assessment

Two observers read all the articles and retrieved the data. These observers were not masked for the journal or any other aspect of the article. Data on inclusion criteria, exclusion criteria and adverse events were copied from a HTLM version of the article and edited for the table. One other observer was given only the text of the method section to score quality items. This observer was also given a separate results section to score the baseline and change in ETDRS score and CRT. This observer was blinded for the results of the other observers. Differences between observers were discussed until a consensus was reached. The items scored for quality of the follow-up studies were: prospective followup, consecutive case series, loss to follow-up, masked assessment of the outcome, standardized assessment of the outcome and other interventions during the study period. [39] The baseline value of the VA and CRT and these scores at last examination or at the examination with complete follow-up, when possible, were noted.

Study characteristics and outcome

Data included were author and year, type of study and quality issues, inclusion criteria, exclusion criteria, details of the treatment (systemic or intravitreal therapy, dosage, frequency of dosing and criteria for additional injections), number of patients at baseline and at follow-up and followup time, baseline ETDRS and CRT and changes in these outcomes and adverse events. The quality of the beforeand-after studies was scored according to the following criteria often used to score quality of follow-up studies: prospective study, consecutive cases, loss to follow-up, blind assessment of outcome, standardized assessment of outcome. The criterion "no other intervention" was omitted, since studies that included such intervention were omitted from inclusion.

The quality of the RCTs was scored with the Delphi list, supplemented with criteria from the Dutch Cochrane Centre. The complete list of criteria is shown in Table 2 [44].

\section{Quantitative data analysis}

The VA score was converted to the ETDRS score when the ETDRS score was not assessed directly. We used a 
conversion of 0.1 logmar (one Snellen line) to 5 ETDRS letters (one ETDRS line). The change in ETDRS score was calculated as the difference between baseline value and the value at the last examination, or at the examination when there was no loss to follow-up. A summary for change in ETDRS and CRT was calculated as the weighted mean of the changes. The number of patients at the follow-up examination determined the weight. Calculations were made separately for intravenous injections and intravitreal injections. The RCT arms that included the treatment with only bevacizumab were included in the before-and-after studies. A subgroup analysis was conducted separately for several characteristics for the follow-up studies, which included the following: total quality score, dosage of intravitreal bevacizumab, previous treatment in more than $50 \%$ of patients, size of the study, duration of follow-up, and method of assessment for visual acuity (ETDRS or Snellen). The study quality items and results of the RCTs were also presented. The results of the RCTs were presented as described after conversion to ETDRS scores.

\section{Results}

The total number of articles retrieved was 561. A large number of articles $(n=535)$ were not included in the review because of several reasons (Table 1). Ultimately, 26 out of these 561 were suitable and used in the analysis. Three randomised controlled trials were published and presented separately $[9,27,31]$. We also combined in this review the data of the patients of these three RCTs who had received

Table 1 Flow chart for the selection of articles

\begin{tabular}{ll}
\hline Number of articles after the search & 56 \\
Reasons for not including an article & \\
Review, comments, guidelines other reasons & 228 \\
Other eye diseases than age-related macular degeneration & 133 \\
Adverse events or safety of bevacizumab as & 59 \\
primary goal of study outcome & 34 \\
Other diseases than eye diseases & 28 \\
Other primary study therapy in AMD & 24 \\
Basic science or experimental studies & 9 \\
Case series & 7 \\
Kinetics and dosage study of anti-vegf & 5 \\
Study on costs & 3 \\
Study conducted to study the differences & \\
between subgroups in effect of bevacizumab & 2 \\
Follow-up with only OCT & 1 \\
Retrospective comparison of bevacizumab & \\
with ranibizumab (abstract only) & 1 \\
Co-interventions given & 1 \\
Not only patients with AMD included & \\
Included in the study & 26 \\
\hline
\end{tabular}

only intravitreal bevacizumab, and the data of patients of 23 before-and-after studies with follow-up of patients who had received bevacizumab for exudative AMD (Tables 2, 3, 4 and 5) [1-4, 7-10, 15-18, 23, 25, 27, 29, 30-35, 37, 40, 41, 48]. A total of 1,435 patients included at baseline in these studies were included in this review.

The three RCTs all showed that bevacizumab is more effective than PDT (with or without triamcinolone) [9, 27, 31]. The RCTs were of a poor-to-reasonable quality and lack of masking was the main methodological problem.

Two articles showed the results of the same study [34, 35]. Of these two, the article with the largest series of patients and longest follow-up period was included in this review [35]. In this study the effect of intravenous bevacizumab in AMD was studied. Two other studies included patients who had received intravenous bevacizumab $[10,23]$. The data of these three studies were included in the summary of the effect of intravenous injections. After intravenous bevacizumab the weighted mean change in ETDRS score was +12.8 (range +11 to +14 ) and the weighted mean change in CRT was $-129 \mu \mathrm{m}$ (range -100 to -202 ).

Twenty-three articles, including the patients in the RCTs who received bevacizumab, reported the change in VA after intravitreal bevacizumab (see Table 2) $[1-4,9,15-18,23,25$, $27-33,37,40,48]$. The weighted mean change after intravitreal injections was 8.6 (range 2 to 26), and for the CRT -90 (range -46 to -190 ). The data are summarised in Tables 5 and 6 . Inclusion criteria varied, and were composed of: age range, VA range, recent disease progression, poor

Table 2 Study quality scores (according to Delphi list supplemented with criteria from the Dutch Cochrane centre) of three randomized controlled clinical trials that included one treatment group with intravitreal bevacizumab

\begin{tabular}{llll}
\hline Study characteristics and outcome & {$[9]$} & {$[27]$} & {$[30]$} \\
\hline Randomization & Yes & Yes & Yes \\
Allocation concealed & Uk & Uk & Uk \\
$\begin{array}{l}\text { Groups similar at baseline regarding } \\
\text { prognostic factors or were }\end{array}$ & Uk & Yes & Yes \\
$\begin{array}{l}\text { adjustments for differences made } \\
\text { in the analysis }\end{array}$ & & & \\
Eligibility criteria specified & Yes & Yes & Yes \\
Other interventions applied & No & No & No \\
Outcome assessor blinded & No & Uk & Yes \\
Care provider blinded & No & No & No \\
Patient blinded & No & No & No \\
Sufficient number with follow-up & Yes & Yes & Yes \\
Point estimates and measures of & No & Yes & Yes \\
$\quad$ variability presented & & & \\
Intention to treat analysis & Yes & Yes & Yes \\
Total quality score & $5 / 11$ & $7 / 11$ & $8 / 11$ \\
\hline Uk: unknown & & &
\end{tabular}

Uk: unknown 
Table 3 Results of three randomized controlled trials that included one group that received intravitreal bevacizumab

\begin{tabular}{lllllll}
\hline Study & Intervention & Number of patients & ETDRS baseline & ETDRS change & CRT base-line & CRT change \\
\hline$[9]$ & Bevacizumab & 32 & 47 & 12 & 354 & -113 \\
& PDT & 30 & 49 & -6 & 352 & -60 \\
{$[27]$} & Bevacizumab & 10 & 54 & 12 & 371 & -138 \\
& PDT + triamcinolone & 10 & 42 & -7 & 346 & -132 \\
& PDT + triamcinolone, reduced & 10 & 43 & $8.6(7.1-10.1)$ & 355 & -78 \\
{$[30]$} & Bevacizumab & 54 & 31 & $2.5(0.9-4.1)$ & 356 & $-55(-64$ to -45$)$ \\
& PDT & 52 & 33 & $12.3(10.1-13.9)$ & 349 & $-54(-64$ to -44$)$ \\
& PDT + Bevacizumab & 52 & & $-65(-74$ to -55$)$ \\
\hline
\end{tabular}

Table 4 Study characteristics of 23 studies on the effect of bevacizumab in age-related exudative AMD

\begin{tabular}{|c|c|c|c|c|c|c|c|}
\hline Study & Inclusion criteria & Prospective & $\begin{array}{l}\text { Consecutive } \\
\text { cases }\end{array}$ & $\begin{array}{l}\text { Loss to } \\
\text { follow-up }\end{array}$ & $\begin{array}{l}\text { Blind } \\
\text { assessment }\end{array}$ & $\begin{array}{l}\text { Standardized } \\
\text { assessment }\end{array}$ & $\begin{array}{l}\text { Previous } \\
\text { treatment }>50 \%\end{array}$ \\
\hline$[34,35]$ & $\begin{array}{l}\text { CNV location: subfoveal } \\
\text { Lesion composition: all lesion types } \\
\text { if recent progression. }\end{array}$ & Yes & No & No & No & Yes & \\
\hline$[10]$ & $\begin{array}{l}\text { CNV location: nn } \\
\text { Lesion composition: pigment } \\
\text { epithelial detachment. }\end{array}$ & Yes & Yes & No & $\begin{array}{l}\text { No (yes } \\
\text { for OCT) }\end{array}$ & Yes & \\
\hline [23] & $\begin{array}{l}\text { CNV location: } \mathrm{nn} \\
\text { Lesion composition: all lesion types. }\end{array}$ & Yes & No & No & No & Yes & \\
\hline$[1]$ & $\begin{array}{l}\text { CNV location: } \mathrm{nn} \\
\text { Lesion composition: all lesion types. }\end{array}$ & Yes & No & No & No & No & No \\
\hline [7] & $\begin{array}{l}\text { CNV location: subfoveal } \\
\text { Lesion composition: all lesion types, except } \\
\text { minimally classic } \mathrm{CNV}>4 \text { disk } \\
\text { areas or those with } \mathrm{CNV} \text { that is more } \\
\text { than } 50 \% \text { obscured by blood }\end{array}$ & Yes & No & No & No & No & No \\
\hline$[23]$ & $\begin{array}{l}\text { CNV location: subfoveal } \\
\text { Lesion composition: all active lesion types. }\end{array}$ & Yes & No & No & No & Yes & No \\
\hline [3] & $\begin{array}{l}\text { CNV location: } \mathrm{nn} \\
\text { Lesion composition: pure or predominantly } \\
\text { occult }+ \text { recent progression. }\end{array}$ & $\mathrm{Nr}$ & Yes & No & No & Yes & No \\
\hline [4] & $\begin{array}{l}\text { CNV location: subfoveal CNV } \\
\text { Lesion composition: all lesion types. }\end{array}$ & No & Yes & Possible & No & No & No \\
\hline$[30]$ & $\begin{array}{l}\text { CNV location: subfoveal lesion } \\
\text { Lesion composition: Minimally classic, occult } \\
\text { with active leakage by FAG and OCT. }\end{array}$ & Yes & No & Possible & No & Yes & No \\
\hline$[37]$ & $\begin{array}{l}\text { CNV location: } \mathrm{nn} \\
\text { Lesion composition: all lesion types }\end{array}$ & No & No & No & No & No & No \\
\hline$[40]$ & $\begin{array}{l}\text { CNV location: subfoveal } \\
\text { Lesion composition: Initially patients who } \\
\text { responded poorly on other treatments, later } \\
\text { large minimally classic or occult CNV. }\end{array}$ & No & Yes & Yes & No & No & No \\
\hline [48] & $\begin{array}{l}\text { CNV location: subfoveal } \\
\text { Lesion composition: all types. }\end{array}$ & No & No & No & No & Yes & No \\
\hline [29] & $\begin{array}{l}\text { CNV location: subfoveal CNV } \\
\text { Lesion composition: all lesion types }\end{array}$ & No & No & No & No & Yes & No \\
\hline$[15]$ & $\begin{array}{l}\text { CNV location: } \mathrm{nn} \\
\text { Lesion composition: all lesion types }\end{array}$ & Yes & Yes & No & No & No & No \\
\hline [2] & CNV location: subfoveal & No & No & Possible & No & No & No \\
\hline
\end{tabular}


Table 4 (continued)

\begin{tabular}{|c|c|c|c|c|c|c|c|}
\hline Study & Inclusion criteria & Prospective & $\begin{array}{l}\text { Consecutive } \\
\text { cases }\end{array}$ & $\begin{array}{l}\text { Loss to } \\
\text { follow-up }\end{array}$ & $\begin{array}{l}\text { Blind } \\
\text { assessment }\end{array}$ & $\begin{array}{l}\text { Standardized } \\
\text { assessment }\end{array}$ & $\begin{array}{l}\text { Previous } \\
\text { treatment }>50 \%\end{array}$ \\
\hline$[41]$ & $\begin{array}{l}\text { Lesion composition: all lesion types } \\
\text { CNV location: some part of the lesion } \\
\text { had to involve the fovea. }\end{array}$ & No & Yes & Yes & No & No & Yes \\
\hline$[27]$ & $\begin{array}{l}\text { Lesion composition: submacular hemorrhage } \\
\text { of }>50 \% \text { of the lesion size. } \\
\text { CNV location: subfoveal } \\
\text { Lesion composition: } 5 \text { occult type, } 2 \text { classic, } \\
3 \text { minimal classic }\end{array}$ & Yes & Yes & Yes & No & Yes & No \\
\hline$[18]$ & $\begin{array}{l}\text { CNV location: subfoveal } \\
\text { Lesion composition: all lesion types }\end{array}$ & No & No & No & No & Yes & Yes \\
\hline [16] & $\begin{array}{l}\text { CNV location: subfoveal } \\
\text { Lesion composition: Refractory pigment } \\
\text { epithelial detachment (PED) and occult CNV }\end{array}$ & No & Yes & Yes & No & Yes & Yes \\
\hline [9] & $\begin{array}{l}\text { CNV location: subfoveal } \\
\text { Lesion composition: Predominantly } \\
\text { classic CNV. }\end{array}$ & Yes & No & Yes & No & Yes & No \\
\hline$[8]$ & $\begin{array}{l}\text { CNV location: Subfoveal } \\
\text { Lesion composition: all types except retinal } \\
\text { angiomatous proliferation (RAP) }\end{array}$ & Yes & No & No & No & Yes & No \\
\hline$[31]$ & $\begin{array}{l}\text { CNV location: subfoveal } \\
\text { Lesion composition: minimally classic } \\
\text { or occult. }\end{array}$ & Yes & No & No & No & Yes & No \\
\hline$[33]$ & $\begin{array}{l}\text { CNV location: Mainly subfoveal } \\
\text { Lesion composition: All lesion types. }\end{array}$ & No & Yes & No & No & Yes & No \\
\hline [17] & $\begin{array}{l}\text { CNV location: subfoveal } \mathrm{CNV} \\
\text { Lesion composition: all types }\end{array}$ & No & Yes & No & No & No & No \\
\hline$[25]$ & $\begin{array}{l}\text { CNV location: } \mathrm{nn} \\
\text { Lesion composition: } \mathrm{nn}\end{array}$ & Yes & No & Yes & No & No & Yes \\
\hline$[32]$ & $\begin{array}{l}\text { CNV location: subfoveal } \\
\text { Lesion composition: all active lesion types }\end{array}$ & Yes & No & No & No & No & No \\
\hline
\end{tabular}

nn: not noted, CNV: choroidal neovascularisation; FAG: fluorescein angiography; OCT: optical coherence tomography.

Table 5 Summary of exclusion criteria of the 23 studies on the effect of bevacizumab in age-related exudative AMD

\begin{tabular}{ll}
\hline $\begin{array}{l}\text { Exclusion criteria mentioned } \\
\text { in the various articles }\end{array}$ & Reference numbers \\
\hline Uncontrolled hypertension & {$[1-4,23,25,34,35,37,41,48]$} \\
Thromboembolic events: & {$[1-4,8,10,23,25,29,34$,} \\
MI, CVA & $35,37,41,48]$ \\
Renal abnormalities & {$[3,10,23,34,35]$} \\
History of bleeding and / or & {$[1,8,23,25,34,35]$} \\
$\quad$ coagulation abnormalities & {$[1,3,8,23,25,34,35]$} \\
Recent or planned surgery & {$[1,7,23]$} \\
Any ocular condition that & \\
$\quad$ may affect vision & {$[1,23,28]$} \\
Recent ocular surgery or laser & {$[8,9,27]$} \\
Prior treatment for CNV & {$[15-17,30,32,33,40]$} \\
No exclusion criteria & \\
mentioned & \\
\hline
\end{tabular}

response to previous treatment including PDT or pegaptanib, not eligible for PDT, previous treatment, and type of CNV. A summary of the various exclusion criteria in these studies is displayed in Table 5. Mostly, intravitreal injections of $1.25 \mathrm{mg}$ bevacizumab were given. The strategy for additional injections differed and two main strategies could be discerned: 1) monthly injections irrespective of the results, or 2 ) additional injections when vision loss reoccurred or when macular fluid reoccurred on OCT. The mean number of patients included at baseline who received intravitreal injections per study was 61 (range 10-266). The weighted mean follow-up time was 15 weeks (range 4-48). The ocular or systemic adverse events that were reported in a total of 1,435 patients who had received an intravitreal injection, and a total of several thousand intravitreal injections, are summarized in Table 6. Table 7 summarizes the incidence of reported presumed adverse events. 
Table 6 Reported results of 23 studies on the effect of bevacizumab in exudative AMD

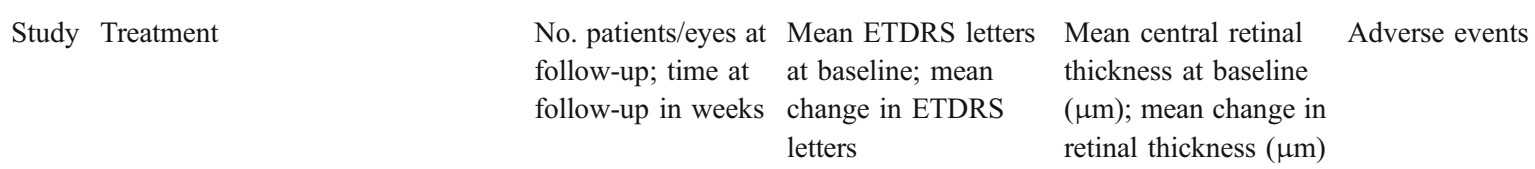

[34, Intravenous. Two to three at 2

35] weeks interval. Additional therapy according to criteria weeks in between

[23] Intravenous. Three with 2 weeks in between

[1] Single intravitreal injection of $2.5 \mathrm{mg}$. No additional injections.
[10] Intravenous. Three with 2

$\begin{array}{lll}n=18 ; 24 \text { weeks } & 54 ;+14 & 392 ;-112 \\ n=9 ; 12 \text { weeks } & 54 ;+12.7 & \text { Not reported; }-202 \\ n=12 ; 12 \text { weeks } & 58.5 ;+11 & 337 ;-100 \\ n=39 ; 4 \text { weeks } & 29 \text { (median); +15 } & 388 \text { (median); }-141\end{array}$

$\begin{array}{lll}n=17 ; 12 \text { Weeks } & 31 ;+26 & 362 ;-151 \\ n=12 ; 12 \text { weeks } & 46.6 ;+8.3 & 402 ;-154 \\ n=30 ; 14 \text { weeks } & 46 ;+10 & 420 \text { (median); }-190 \\ & & \\ n=50 ; 8 \text { weeks } & 36 ;+10 & \text { not reported; }-92 \\ & & 378, \text { change at } \\ n=80 ; 12 \text { weeks } & 32,+8 & 18 \text { weeks }-56 \\ \text { (range } 6-24) & & 351 ;-99.6 \\ n=50 ; 12 \text { weeks } & 38.1 ;+7.9 & 340 ;-127 \\ n=141 ; 12 \text { weeks } & 38 ;+11 & \end{array}$

[40] Single intravitreal injection of $1.25 \mathrm{mg}$. Additional injections according to criteria

[7] Three monthly intravitreal injections of $2.5 \mathrm{mg}$. No additional injections

[23] Three monthly intravitreal injections of $1 \mathrm{mg}$. No additional injections. $1.25 \mathrm{mg}$. Additional injections according to criteria

[4] Single intravitreal injection of $1.25 \mathrm{mg}$. Additional injections according to criteria

[30] Single intravitreal injection of $1.25 \mathrm{mg}$. Additional injections according to criteria

[37] Single intravitreal injection of $1.25 \mathrm{mg}$. Additional injections according to criteria
[3] Single intravitreal injection of
[48] Single intravitreal injection of $1.25 \mathrm{mg}$. Additional injections according to criteria

[29] Single intravitreal injection of $1.25 \mathrm{mg}$ or $1.5 \mathrm{mg}$. Additional $n=80 ; 12$ week

$48.5 ;+8.6$

$337 ;-93$
No serious ocular or systemic adverse events. Increase in systolic and diastolic blood pressure.

No serious ocular or systemic events.

No severe adverse events

A mild inflammatory response in the anterior chamber was the most common side effect observed in eight eyes (20.5\%), followed by subconjunctival haemorrhage in 3 patients

No serious ocular or systemic events

No severe adverse events

No serious ocular or systemic events.

No serious ocular or systemic events.

RIP in two patients. Ten PVD. No serious ocular or systemic events.

No serious ocular or systemic events.

At 1 month, two patients had mild vitritis, and one patient at 2 months, who had a history of recurrent uveitis. No endophthalmitis, increased intraocular pressure, retinal tear, or retinal detachment occurred. One patient had MI after third injection, one treated for TIA stopped anti-coagulants by himself and developed TIA. Another possible TIA, One death of MI (smoker with emfysema).

No serious ocular or systemic events

No serious ocular or systemic events. 
Table 6 (continued)

Study Treatment

$\begin{array}{llll}\text { No. patients/eyes at } & \text { Mean ETDRS letters } & \text { Mean central retinal } & \text { Adverse events } \\ \text { follow-up; time at } & \text { at baseline; mean } & \text { thickness at baseline } & \\ \text { follow-up in weeks } & \begin{array}{l}\text { change in ETDRS } \\ \text { letters }\end{array} & \begin{array}{l}(\mu \mathrm{m}) \text {; mean change in } \\ \text { retinal thickness }(\mu \mathrm{m})\end{array}\end{array}$

injections according to criteria

[15] Single intravitreal injection of $1.25 \mathrm{mg}$. Additional injections

$\begin{array}{lll}n=102 ; 10 \text { weeks } & 56 ;+10 & 251 ;-46 \\ \begin{array}{l}n=17 ; 18 \text { weeks } \\ \text { (mean follow-up) }\end{array} & \text { Not reported; }+5.5 & 404 ;-124 \\ n=21,16 \text { weeks } & 55 ;+4 & 299 ;-62 \\ & & \\ n=10,12 \text { weeks } & 54 ;+12 & 371 ;-138 \\ & & \\ n=68,12 \text { weeks } & 51 ;+4 & 304 ;-68\end{array}$

[18] Single intravitreal injection of $1.25 \mathrm{mg}$. Additional injections according to criteria

[16] Single intravitreal injection of $1.25 \mathrm{mg}$. Additional injections according to criteria

[9] Three monthly intravitreal injections of $2.5 \mathrm{mg}$ bevacizumab. Additional injections according to criteria

[8] Three monthly intravitreal injections of $2.5 \mathrm{mg}$ bevacizumab. Additional injections according to criteria.

[31] Single injection with $1.25 \mathrm{mg}$ bevacizumab. No repeated injections.

[33] Single intravitreal injection of $1.25 \mathrm{mg}$. Additional injections according to criteria.

$n=32,24$ weeks $\quad 47 ;+12 \quad 354 ;-113$

$n=51,48$ weeks $\quad 45.7 ;+7.4 \quad 327 ;-100$

$n=54,12$ weeks $\quad 31 ;+8.6 \quad 355 ;-55$
No adverse ocular or systemic events.

No serious ocular or systemic events.

No serious ocular or systemic adverse events

No serious ocular or systemic adverse events

No serious injection-related adverse events.

$n=10,16$ weeks $\quad 21 ;+15 \quad$ Not quantified

No systemic or ocular adverse events

No systemic or ocular adverse events

No serious ocular or systemic events.

Three pigment epithelial ruptures, 12 PVD, four cataract progression. Some patients reported blurred vision during a few days. One case of endophthalmitis. One patient developed a retinal pigment epithelial rip 2 weeks after treatment. No patient had thromboembolism or any other systemic adverse events.

[17] Single intravitreal injection of $n=103,8$ weeks $\quad 44 ;+6$

CRT not in every patient conducted according to criteria.

[25] Three monthly intravitreal injections of $1.25 \mathrm{mg}$ $n=27,12$ weeks $\quad 50 ;+2$ 373;-94 bevacizumab.

[32] Single intravitreal injection of $\mathrm{N}=48 ; 15.4$ weeks $\quad 32 ;+8$ $389 ;-51$
One case of endophthalmitis, three submacular hemorrhages, and three RPE tears.

Two transient corneal epitheliopathy. Two patients discontinued treatment due to RPE tears. No other systemic or ocular adverse events.

No adverse ocular or systemic events. 
Table 7 Reported adverse events in the 23 follow-up studies with intravitreal injections of bevacizumab for exudative AMD, in a total of 1,396 patients who received one or more injections

\begin{tabular}{lll}
\hline Event & Number of reported case & $\begin{array}{l}\text { Cumulative } \\
\text { incidence per } \\
100 \text { patients }\end{array}$ \\
& & \\
\hline Posterior vitreous detachment & 24 & 1.7 \\
Pigment epithelial rupture & 15 & 1.1 \\
Vitritis/uveitis & 8 & 0.56 \\
Subconjunctival hemaorrhage & 3 & 0.21 \\
Submacular hemaorrhage & 3 & 0.21 \\
Cataract progression & 4 & 0.18 \\
Endophthalmitis & 2 & 0.14 \\
Corneal epitheliopahty & 2 & 0.14 \\
Myocardial Infarction (MI)I & 2 (one 88 years old, 2 months after third injection); \\
TIA & 1 (stopped anti-coagulants himself) & 0.14 \\
MI death & 1 (smoker with emphysema) & 0.07 \\
CVA & 1 (83 years old, 34 days after second injection) & 0.07 \\
Blurred vision during few days & Some patients & 0.07 \\
\hline
\end{tabular}

Studies differed in quality (Tables 4 and 8). The difference in change in VA between subgroups according to differences in quality was no more than 1.9 letters (Table 8). The change in ETDRS score was 2.7 letters higher for the studies with a higher quality. No study had a blinded assessment of the outcome, except for one study (but blinded only for the assessment of the CRT [10]. The highest difference between two groups was for those who had a higher dosage compared to those with a lower dosage (Table 8).

\section{Discussion}

This systematic review shows that after administration of bevacizumab in patients with exudative age-related macular

Table 8 Change in ETDRS score and central retinal thickness after intravitreal bevacizumab stratified according to different study characteristics

\begin{tabular}{|c|c|c|c|c|c|c|c|}
\hline $\begin{array}{l}\text { Study } \\
\text { characteristic }\end{array}$ & $\begin{array}{l}\text { Value of } \\
\text { study } \\
\text { characteristic }\end{array}$ & $\begin{array}{l}\text { Number of patients } \\
\text { for ETDRS change } \\
\text { calculation }\end{array}$ & $\begin{array}{l}\text { ETDRS } \\
\text { change }\end{array}$ & $\begin{array}{l}\text { ETDRS difference } \\
\text { between the two } \\
\text { groups }\end{array}$ & $\begin{array}{l}\text { Number of patients } \\
\text { included for CRT } \\
\text { change calculation }\end{array}$ & $\begin{array}{l}\text { CRT } \\
\text { Change }\end{array}$ & $\begin{array}{l}\text { CRT difference } \\
\text { between the } \\
\text { two groups }\end{array}$ \\
\hline \multirow[t]{2}{*}{ Prospective study } & Yes & 494 & 9.7 & \multirow[t]{2}{*}{1.9} & 494 & 78 & \multirow[t]{2}{*}{-24} \\
\hline & No & 661 & 7.8 & & 548 & 102 & \\
\hline \multirow{2}{*}{$\begin{array}{l}\text { Consecutive inclusion } \\
\text { of patients }\end{array}$} & Yes & 510 & 9.0 & \multirow[t]{2}{*}{0.7} & 397 & 99 & \multirow[t]{2}{*}{14} \\
\hline & No & 645 & 8.3 & & 645 & 85 & \\
\hline \multirow[t]{2}{*}{ Loss to follow-up } & Yes & 478 & 7.9 & \multirow[t]{2}{*}{-0.4} & 677 & 86 & \multirow[t]{2}{*}{-10} \\
\hline & No & 677 & 9.7 & & 478 & 96 & \\
\hline \multirow{2}{*}{$\begin{array}{l}\text { Standardized assessment } \\
\text { of visual acuity }\end{array}$} & Yes & 540 & 7.8 & \multirow{2}{*}{-1.5} & 530 & 86 & \multirow[t]{2}{*}{-9} \\
\hline & No & 615 & 9.3 & & 512 & 95 & \\
\hline \multirow[t]{2}{*}{ Quality score $\dagger$} & $\geq 4$ out of 5 & 196 & 10.9 & \multirow[t]{2}{*}{2.7} & 186 & 92 & \multirow[t]{2}{*}{2} \\
\hline & $<4$ out of 5 & 959 & 8.2 & & 856 & 90 & \\
\hline \multirow[t]{2}{*}{ Dosage } & $\geq 2 \mathrm{mg}$ & 139 & 13.3 & \multirow[t]{2}{*}{5.3} & 139 & 120 & \multirow[t]{2}{*}{34} \\
\hline & $<2 \mathrm{mg}$ & 1016 & 8.0 & & 903 & 86 & \\
\hline \multirow[t]{2}{*}{ Study size } & $>50$ patients & 801 & 8.4 & \multirow[t]{2}{*}{-0.8} & 344 & 83 & \multirow[t]{2}{*}{-22} \\
\hline & $\leq 50$ patients & 354 & 9.2 & & 698 & 105 & \\
\hline \multirow[t]{2}{*}{ Follow-up duration } & $\geq 12$ weeks & 790 & 8.0 & \multirow[t]{2}{*}{-1.9} & 780 & 95 & \multirow[t]{2}{*}{17} \\
\hline & $<12$ weeks & 365 & 9.9 & & 262 & 78 & \\
\hline \multirow[t]{2}{*}{ Previous treatment } & $<50 \%$ of patients & 621 & 9.0 & \multirow[t]{2}{*}{0.8} & 518 & 93 & \multirow[t]{2}{*}{5} \\
\hline & $\geq 50 \%$ of patients & 534 & 8.2 & & 524 & 88 & \\
\hline \multirow[t]{2}{*}{ Method of VA assessment } & ETDRS & 530 & 7.2 & \multirow[t]{2}{*}{-2.6} & 530 & 81.6 & \multirow[t]{2}{*}{-1.2} \\
\hline & Snellen & 625 & 9.8 & & 615 & 82.8 & \\
\hline
\end{tabular}

$\dagger$ as defined by the presence of $>4$ out of 5 of the following: prospective follow-up, consecutive case series, loss to follow-up, masked assessment of the outcome, standardized assessment of the outcome 
degeneration, an improvement in both the VA and CRT are reported. Moreover, bevacizumab is more efficacious than PDT. The weighted mean increase in VA after intravitreal bevacizumab injections in 1,396 patients is 8.6 letters on the ETDRS score. The weighted mean decrease in CRT is $90 \mu \mathrm{m}$. The incidence of adverse events was low. All studies showed an impressive effect, and the three RCTs showed superiority of bevacizumab to PDT with or without intravitreal triamcinolon.

The observed change in the follow-up studies of bevacizumab is similar to the change observed after monthly intravitreal injections of ranibizumab in exudative age-related macular degeneration. The mean change in ETDRS letters after monthly injections of $0.5 \mathrm{mg}$ ranibizumab varied between +5.9 for occult or minimally classic $\mathrm{CNV}$ and +9.8 for classic $\mathrm{CNV}$ after 3 months $[13,38]$. The observed increase in VA is also closely similar to the increase of 10.8 ETDRS letters after ranibizumab three times and additional injections when necessary based on OCT and VA change [21]. It is interesting to see that in the 1-year follow-up study of Bashur et al., fewer injections were needed than in the 1-year follow-up study on ranibizumab of Fung et al., with almost the same criteria for reinjection, knowing that the intravenous half-life of bevacizumab is $150 \%$ that of ranibizumab in rabbit eyes [5, $6,8,21]$. However, the dosage in Bashur's study was $2.5 \mathrm{mg}$ bevacizumab.

The similarity in effect between bevacizumab and ranibizumab is also supported by a non-randomised, single centre comparative study in which these two drugs were compared [42]. Baseline patient characteristics were similar in both groups of 44 eyes in the bevacizumab group and the 53 eyes in the ranibizumab group. After 113 days of followup and a mean of 1.8 injections in the bevacizumab group the mean increase in VA was 0.8 lines. After 44 days of follow-up and a mean of 2.0 injections of ranibizumab, the mean increase in VA was 0.7 lines $(p=0.87)$. The improvement in VA is also observed in a study that compared starting with bevacizumab to starting with pegaptanib followed by bevacizumab [19]. In the latter group, no statistically significant difference from baseline was observed.

Adverse events were rare in the 1,396 patients who received a total of several thousand intravitreal injections. Moreover, some adverse events are the result of the procedure (endophthalmitis, retinal detachment, cataract progression), others are mild (vitritis), or an event may also occur as a result of the natural course of AMD itself or with other therapies such as photodynamic therapy and ranibizumab [14, 24]. Although the reported thromboembolic events could have been caused by bevacizumab, the time frame between injection and the event, the presence of additional risk factors, the age of the population, or the increased risk of cardiovascular diseases in patients with AMD make this less likely. On the other hand, patients at risk of a thromboembolic event were excluded in some studies.

These results confirm the results of an internet surveillance program in which the follow-up for adverse events in 5,228 patients and 7,113 intravitreal injections with bevacizumab from 70 centres in 12 countries was reported [20]. Adverse events reported were corneal abrasion, lens injury, endophthalmitis, retinal detachment, inflammation or uveitis, cataract progression, acute vision loss, central retinal artery occlusion, subretinal haemorrhage, retinal pigment epithelium tears, blood-pressure elevation, transient ischaemic attack, cerebrovascular accident and death. None of the adverse event rates exceeded $0.21 \%$ [22]. These results are also confirmed by a 12-month follow-up study of 1,265 consecutive patients with various diagnoses, including exudative AMD, who received 4,303 intravitreal injections of bevacizumab [46]. Systemic adverse events occurred in eighteen patients $(1.5 \%)$. These were: acute elevation of blood pressure $(0.59 \%)$, cerebrovascular accidents $(0.5 \%)$, myocardial infarction $(0.4 \%)$, iliac artery aneurysms $(0.17 \%)$, toe amputation $(0.17 \%)$ and deaths $(0.4 \%)$. Ocular adverse events were bacterial endophthalmitis $(0.16 \%)$, tractional retinal detachment $(0.16 \%)$, uveitis $(0.09 \%)$, rhegmatogenous retinal detachment $(0.02 \%)$ and vitreous haemorrhage. Some of the adverse events may have been the result of the underlying disease. The type and incidence of the reported adverse events in the bevacizumab studies does not seem to be very different from the reported type and incidence from two large RCTs of ranibizumab $[12,38]$. The frequency of injections in the two ranibizumab RCTs was monthly for a period of 1 year, while in the studies included in this review only a single intravitreal injection, or three monthly injections with or without repeated injections were given. This treatment will ultimately lead to fewer injectionrelated adverse events in the latter case.

It is known that Snellen and ETDRS visual acuities don't match exactly. One shortcoming of this review, therefore, was that Snellen acuities as reported in the various studies were converted to ETDRS scores using a conversion of 0.1 logmar (one Snellen line) to 5 ETDRS letters (one ETDRS line). On the other hand, for patients with macular degeneration in the lower vision range, it was recently shown that visual acuity measurement with Snellen charts may underestimate VA as compared to measurements with ETDRS charts [19]. This implies that the visual acuity results of this systematic review, which included several studies using Snellen charts only, may underestimate visual acuity improvement after bevacizumab as compared to the ETDRS assessments in the ranibizumab trials. [20]

We conclude that bevacizumab is effective in improving VA in exudative AMD, and that its effect is likely to 
be equivalent to ranibizumab. According to current reports severe adverse events for bevacizumab are rare in the short term.

Open Access This article is distributed under the terms of the Creative Commons Attribution NonCommercial License which permits any noncommercial use, distribution, and reproduction in any medium, provided the original author(s) and source are credited.

\section{References}

1. Abraham Marin ML, Cortes Luna CF, Alvarez Rivera G, Hernandez Rojas M, Quiroz Mercado H, Morales Canton V (2006) Intravitreal bevacizumab therapy for neovascular agerelated macular degeneration: a pilot study. Graefes Arch Clin Exp Ophthalmol 245:651-655, doi:10.1007/s00417-006-0411-6

2. Aggio FB, Farah ME, Silva WC, Melo GB (2006) Intravitreal bevacizumab for exudative age-related macular degeneration after multiple treatments. Graefes Arch Clin Exp Ophthalmol 245:215220, doi:10.1007/s00417-006-0412-5

3. Aisenbrey S, Ziemssen F, Volker M, Gelisken F, Szurman P, Jaissle $G$ et al (2006) Intravitreal bevacizumab (Avastin) for occult choroidal neovascularization in age-related macular degeneration. Graefes Arch Clin Exp Ophthalmol 245:941-948, doi:10.1007/ s00417-006-0471-7

4. Avery RL, Pieramici DJ, Rabena MD, Castellarin AA, Nasir MA, Giust MJ (2006) Intravitreal bevacizumab (Avastin) for neovascular age-related macular degeneration. Ophthalmology 113:363-372, doi:10.1016/j.ophtha.2005.11.019

5. Bakri SJ, Snyder MR, Reid JM, Pulido JS, Ezzat MK, Singh RJ (2007) Pharmacokinetics of intravitreal ranibizumab (Lucentis). Ophthalmology 114:2179-2182, doi:10.1016/j.ophtha.2007.09.012

6. Bakri SJ, Snyder MR, Reid JM, Pulido JS, Singh RJ (2007) Pharmacokinetics of intravitreal bevacizumab (Avastin). Ophthalmology 114:855-859, doi:10.1016/j.ophtha.2007.01.017

7. Bashshur ZF, Bazarbachi A, Schakal A, Haddad ZA, El Haibi CP, Noureddin BN (2006) Intravitreal bevacizumab for the management of choroidal neovascularization in age-related macular degeneration. Am J Ophthalmol 142:1-9, doi:10.1016/j. ajo.2006.02.037

8. Bashshur ZF, Haddad ZA, Schakal A, Jaafar RF, Saab M, Noureddin BN (2008) Intravitreal bevacizumab for treatment of neovascular age-related macular degeneration: a one-year prospective study. Am J Ophthalmol 145:249-256, doi:10.1016/j. ajo.2007.09.031

9. Bashshur ZF, Schakal A, Hamam RN, El-Haibi CP, Jaafar RF, Noureddin BN (2007) Intravitreal bevacizumab vs verteporfin photodynamic therapy for neovascular age-related macular degeneration. Arch Ophthalmol 125:1357-1361, doi:10.1001/ archopht.125.10.1357

10. Bolz M, Michels S, Geitzenauer W, Prager F, Schmidt Erfurth U (2006) Effect of systemic bevacizumab therapy on retinal pigment epithelial detachment. Br J Ophthalmol 91:785-789, doi:10.1136/ bjo.2006.102467

11. Bressler NM, Bressler SB, Congdon NG, Ferris FLr, Friedman DS, Klein R, Lindblad AS, Milton RC, Seddon JM, Age-Related Eye Disease Study Research Group (2003) Potential public health impact of age-related eye disease study results: AREDS report no. 11. Arch Ophthalmol 121:1621-1624, doi:10.1001/archopht. 121.11.1621

12. Brown DM, Kaiser PK, Michels M, Soubrane G, Heier JS, Kim RY, Sy JP, Schneider S, ANCHOR-Study-Group (2006) Ranibizumab versus verteporfin for neovascular age-related macular degeneration. N Engl J Med 355:1432-1444, doi:10.1056/NEJMoa062655

13. Brown MM, Brown GC, Sharma S, Stein JD, Roth Z, Campanella J et al (2006) The burden of age-related macular degeneration: a valuebased analysis. Curr Opin Ophthalmol 17:257-266

14. Carvounis PE, Kopel AC, Benz MS (2007) Retinal pigment epithelial tears following ranibizumab for exudative age-realted macualr degeneration. Am J Ophthalmol 143:504-505, doi:10.1016/j.ajo.2006.11.028

15. Chen CY, Wong TY, Heriot WJ (2007) Intravitreal bevacizumab (Avastin) for neovascular age-related macular degeneration: a short-term study. Am J Ophthalmol 143:510-512, doi:10.1016/j. ajo.2006.10.004

16. Chen E, Kaiser RS, Vander JF (2007) Intravitreal bevacizumab for refractory pigment epithelial detachment with occult choroidal neovascularization in age-related macular degeneration. Retina 27 (4):445-450

17. Cleary CA, Jungkim S, Ravikumar K, Kelliher C, Acheson RW, Hickey-Dwyer M (2008) Intravitreal bevacizumab in the treatment of neovascular age-related macular degeneration, 6- and 9-month results. Eye 22:82-86, doi:10.1038/sj.eye.6702936

18. Emerson MV, Lauer AK, Flaxel CJ, Wilson DJ, Francis PJ, Stout JT et al (2007) Intravitreal bevacizumab (Avastin) treatment of neovascular age-related macular degeneration. Retina 27:439-444, doi:10.1097/IAE.0b013e31804b3e15

19. Falkenstein IA, Cheng L, Morrison VL, Kozak I, Tammewar AM, Freeman WR (2007) Standardized visual acuity results associated with primary versus secondary bevacizumab (avastin) treatment for choroidal neovascularization in age-related macular degeneration. Retina 27:701-706, doi:10.1097/ IAE.0b013e3180654240

20. Falkenstein IA, Cochran DE, Azen SP, Dustin L, Tammewar AM, Kozak I et al (2008) Comparison of visual acuity in macular degeneration patients measured with Snellen and early treatment diabetic retinopathy study charts. Ophthalmology 115:319-323

21. Fung AE, Lalwani GA, Rosenfeld PJ, Dubovy SR, Michels S, Feuer WJ, Puliafito CA, Davis JL, Flynn HW, Esquiabro M (2007) An optical coherence tomography-guided, variable dosing regimen with intravitreal ranibizumab (Lucentis) for neovascular age-related macular degeneration. Am J Ophthalmol 143:566-583

22. Fung AE, Rosenfeld PJ, Reichel E (2006) The International Intravitreal Bevacizumab Safety Survey: Using the internet to assess drug safety worldwide. Br J Ophthalmol 90:1344-1349, doi:10.1136/bjo.2006.099598

23. Geitzenauer W, Michels S, Prager F, Kornek G, Vormittag L, Rosenfeld P et al (2006) Early effects of systemic and intravitreal bevacizumab (AvastinR) therapy for neovascular age-related macular degeneration. Klin Monatsblat Augenheilkund 223:822827, doi:10.1055/s-2006-926875

24. Gelisken F, Inhoffen W, Partsch M, Schneider U, Kreissig I (2001) Retinal pigment epithelial tear after photodynamic therapy for choroidal neovascularization. Am J Ophthalmol 131:518-520, doi:10.1016/S0002-9394(00)00813-8

25. Giansanti F, Virgili G, Bini A, Rapizzi E, Giacomelli G, Donati MC et al (2007) Intravitreal bevacizumab therapy for choroidal neovascularization secondary to age-related macular degeneration: 6month results of an open-label uncontrolled clinical study. Eur J Ophthalmol 17:230-237

26. Gragoudas ES, Adamis AP, Cunningham ET Jr, Feinsod M, Guyer DR, VEGF-Inhibition-Study-in-Ocular-NeovascularizationClinical-Trial-Group (2004) Pegaptanib for neovascular agerelated macular degeneration. N Engl J Med 351:2805-2816, doi:10.1056/NEJMoa042760

27. Hahn R, Sacu S, Michels S, Varga A, Weigert G, Geitzenauer W et al (2007) [Intravitreal bevacizumab versus verteporfin and intra- 
vitreal triamcinolone acetonide in patients with neovascular agerelated macula degeneration]. Ophthalmologe 104(7):588-593

28. Kliffen M, Sharma HS, Mooy CM, Kerkvliet S, de-Jong PT (1997) Increased expression of angiogenic growth factors in agerelated maculopathy. Br J Ophthalmol 81:154-162

29. Ladewig MS, Ziemssen F, Jaissle G, Helb HM, Scholl HPN, Eter N et al (2006) Intravitreal bevacizumab for neovascular age-related macular degeneration. Ophthalmologe 103:463-470, doi:10.1007/ s00347-006-1352-5

30. Lazic R, Gabric N (2007) Intravitreally administered bevacizumab (Avastin) in minimally classic and occult choroidal neovascularization secondary to age-related macular degeneration. Graefes Arch Clin Exp Ophthalmol 245:68-73, doi:10.1007/s00417-006-0466-4

31. Lazic R, Gabric N (2007) Verteporfin therapy and intravitreal bevacizumab combined and alone in choroidal neovascularization due to age-related macular degeneration. Ophthalmology 114:1179-1185, doi:10.1016/j.ophtha.2007.03.006

32. Lazic R, Gabric N, Dekaris I, Saric B, Gavric M (2007) Intravitreal bevacizumab (Avastin) in treatment of neovascular age-related macular degeneration. Coll Antropol 31:77-81

33. Madhusudhana KC, Hannan SR, Williams CP, Goverdhan SV, Rennie C, Lotery AJ et al (2007) Intravitreal bevacizumab (Avastin) for the treatment of choroidal neovascularization in age-related macular degeneration: results from 118 cases. $\mathrm{Br} \mathrm{J}$ Ophthalmol 91:1716-1717, doi:10.1136/bjo.2006.108639

34. Michels S, Rosenfeld PJ, Puliafito CA, Marcus EN, Venkatraman AS (2005) Systemic bevacizumab (Avastin) therapy for neovascular agerelated macular degeneration: twelve-week results of an uncontrolled open-label clinical study. Ophthalmology 112:1035-1047, doi:10.1016/j.ophtha.2005.02.007

35. Moshfeghi AA, Rosenfeld PJ, Puliafito CA, Michels S, Marcus EN, Lenchus JD, Venkatraman AS (2006) Systemic bevacizumab (Avastin) therapy for neovascular age-related macular degeneration: twenty-four-week results of an uncontrolled open-label clinical study. Ophthalmology 113:2002 e2001-2012

36. Mulcahy MF, Benson AB (2005) Bevacizumab in the treatment of colorectal cancer. Expert Opin Biol Ther 5:997-1005, doi:10.1517/14712598.5.7.997

37. Rich RM, Rosenfeld PJ, Puliafito CA, Dubovy SR, Davis JL, Flynn HW et al (2006) Short-term safety and efficacy of intravitreal bevacizumab (Avastin) for neovascular age-related macular degeneration. Retina 26:495-511, doi:10.1097/01. iae.0000225766.75009.3a

38. Rosenfeld PJ, Brown DM, Heier JS, Boyer DS, Kaiser PK, Chung CY, Kim RY, MARINA Study Group (2006) Ranibizumab for neovascular age-related macular degeneration. N Engl J Med 355:1419-1431, doi:10.1056/NEJMoa054481
39. Sackett DL, Haynes RB, Guyatt GH, Tugwell P (1991) Clinical epidemiology. A basic science for clinical medicine. Little, Brown and Company, Boston

40. Spaide RF, Laud K, Fine HF, Klancnik JM Jr, Meyerle CB, Yannuzzi LA et al (2006) Intravitreal bevacizumab treatment of choroidal neovascularization secondary to age-related macular degeneration. Retina 26:383-390, doi:10.1097/00006982200604000-00001

41. Stifter E, Michels S, Prager F, Georgopoulos M, Polak K, Hirn C et al (2007) Intravitreal bevacizumab therapy for neovascular age-related macular degeneration with large submacular hemorrhage. Am J Ophthalmol 144:886-892, doi:10.1016/j.ajo. 2007.07.034

42. Sund NJ, Lai MM, Capone A Jr et al (2007) Efficacy of intravitreal bevacizumab (AvastinTM) vs ranibizumab (LucentisTM) as first-line monotherapy for the treatment of neovascular age-related macular degeneration. Invest Ophthalmol Vis Sci 48: E-Abstract 1813

43. Takeda AL, Colquitt J, Clegg AJ, Jones J (2007) Pegabtanib and ranibizumab for neovascular age-related macular degeneration: a systematic review. Br J Ophthalmol 91:1177-1182, doi:10.1136/ bjo.2007.118562

44. Verhagen AP, de Vet HC, de Bie RA, Kessels AG, Boers M, Bouter LM et al (1998) The Delphi list: a criteria list for quality assessment of randomized clinical trials for conducting systematic reviews developed by Delphi consensus. J Clin Epidemiol 51:1235-1241, doi:10.1016/S0895-4356(98)00131-0

45. VISION Clinical Trial Group (2006) Year 2 efficacy results of 2 randomized controlled clinical trials of pegaptanib for neovascular age-related macular degeneration. Ophthalmology 113:1508-1521

46. Wu L, Martinez-Castellanos MA, Quiroz-Mercado H, Arevalo JF, Berrocal MH, Farah ME, Maia M, Roca JA, Rodriguez FJ, Pan-American Collaborative Retina Study Group (Pacores) (2007) Twelve-month safety of intravitreal injections of bevacizumab (Avastin): results of the Pan-American Collaborative Retina Study Group (Pacores). Graefes Arch Clin Exp Ophthalmol 246(1):81-87

47. Yang JC, Haworth L, Sherry RM, Hwu P, Schwartzentruber DJ, Topalian SL et al (2003) A randomized trial of bevacizumab, an anti-vascular endothelial growth factor antibody, for metastatic renal cancer. N Engl J Med 349:427-434, doi:10.1056/ NEJMoa021491

48. Yoganathan P, Deramo VA, Lai JC, Tibrewala RK, Fastenberg DM (2006) Visual improvement following intravitreal bevacizumab (Avastin) in exudative age-related macular degeneration. Retina 26:994-998, doi:10.1097/01.iae.0000244380.34082.67 